\title{
An Analysis of Physician Productivity and Self-Sustaining Revenue Generation in a Free-Standing Emergency Department Medical Scribe Model
}

\author{
Brody Dawkins, Komal N. Bhagudas, Joshua Hurwitz, J. Adrian Tyndall, \\ Marcelo E. Guerra, Michael Falgiani, Thomas F. Payton, and Brandon R. Allen \\ Department of Emergency Medicine, 1329 SW 16th Street, P.O. Box 100186, Gainesville, FL 32610-0186, USA
}

Correspondence should be addressed to Brandon R. Allen; brandonrallen@ufl.edu

Received 29 August 2015; Revised 5 December 2015; Accepted 7 December 2015

Academic Editor: Frank Hildebrand

Copyright (C) 2015 Brody Dawkins et al. This is an open access article distributed under the Creative Commons Attribution License, which permits unrestricted use, distribution, and reproduction in any medium, provided the original work is properly cited.

\begin{abstract}
Objectives. A free-standing emergency department (FSED) is a facility that provides comprehensive emergency medical care similar to a traditional emergency department but is not attached to a hospital campus. Medical scribes are increasingly likely to work in free-standing emergency departments. The purpose of this study was to retrospectively investigate the benefits of a scribe program in an FSED. Methods. A retrospective, Institutional Review Board-approved analysis from December 1, 2013, to February 1, 2015, of free-standing emergency department medical data was extracted to determine if scribed charts resulted in increased revenue and improved throughput. Results. When scribes are present in the FSED there is a small, but statistically significant, decrease in time from patient arrival to provider by 2.74 minutes. Scribed charts collected $\$ 4.69$ more per chart and resulted in an increase in productivity. Incremental cost effectiveness ratios resulted in proven cost-utility with a net-positive effect. Conclusion. While there are some gains in terms of operational metrics and provider productivity with the addition of scribes to a free-standing emergency department, there is a net-positive financial impact of scribes. Implementing a scribe program at a FSED is cost-effective and justified from both an operational and a financial analysis.
\end{abstract}

\section{Introduction}

Emergency departments (EDs) throughout the United States have been early adopters of electronic health records in efforts to increase the accuracy, availability, storage, and retrieval of health information in order to provide timely, effective, and efficient patient care. In 2009, one of the key provisions of the American Recovery and Reinvestment Act was a mandate that all public and private health care providers and eligible professionals demonstrate meaningful use of electronic medical records (EMRs). This provision alone has spawned the rapid and widespread adoption of EMRs. Although there has been significant evidence demonstrating the benefits of a systematic approach to collecting and sharing health data on patient care quality outcomes, the EMR has so far proven to be less successful when considering the impact on a provider's time, to the detriment of the patientprovider interaction [1]. In the high-stress, high-volume environment of emergency medicine, EMR adoption in early stages has proven to be a particular challenge to efficient patient care. Previous analysis has shown that emergency medicine physicians dedicate nearly $44 \%$ of their shift to data entry and only $28 \%$ in direct patient care [2]. A separate study sponsored by the American College of Emergency Physicians showed that about half of an emergency medicine physicians' time is spent on indirect patient care activities [3].

The resurgence of medical scribes has become one of the key innovative features in emergency medicine that has sought to counteract the impact of EMRs on provider efficiency and has supported the creation of a significant industry in the United States over the last decade. Several recent studies have provided supporting evidence that medical scribes counteract losses in provider productivity in the ED while substantially increasing provider satisfaction. This has also had the impact of increasing efficiency in high volume environments [4]. One study estimated that when 
transitioning from physical or paper documentation to an EMR, facilities have experienced a typical decrease in productivity of approximately 20-30 percent. After implementing scribes, however, additional productivity has been shown to increase by 6.45 percent [5]. Further, in a 2009 study, it was shown that scribes not only were cost-effective but increased revenue per visit by nearly 15 percent due to improvements in coding completion [6]. When examining other measures, scribes have been shown to increase common metrics used to assess physician productivity [1].

The University of Florida Health Emergency Department is a Level 1 Trauma Center that averages nearly 120,000 visits per year across three distinct sites: an adult emergency department, a pediatric emergency department, and its newest addition, a free-standing emergency department (FSED). In May 2013, to meet challenges faced from working within the EMR, University of Florida Health founded its own scribe program and hired its first class of seven scribes. Since then, the scribe program has grown substantially and has yielded measurable benefits in the form of improved throughput and increased provider satisfaction [4]. The newest setting in which the University of Florida Health scribe program operates full time is the Springhill Emergency Department. This is the newest setting in which the scribe program operates full time.

The concept of a FSED is an innovative business model that will accommodate the growing demand for patient care in the United States. FSEDs are affiliated with hospitals, individual physicians, physician groups, or nonphysician entrepreneurs and staffed by board certified physicians. An article published by the Urgent Care Association of America denoted that in 2009 the American Hospital Association accounted 241 centers in 16 states. Currently in the United States there are estimated 400 FSEDs across 40 states [7]. FSEDs provide more available locations for access to patient care in areas with suboptimal medical outreach, notably rural areas, where there is a national trend of full service rural hospitals closing down, leaving absence of care in suburban/rural areas. FSEDs also lessen the overcrowding in the traditional ED setting with a fraction of the waiting time. This model of an ED offers comprehensive emergency care around the clock all year with many additional resources compared to Urgent Care Centers, which are commonly open only 12 hours a day [7]. As stated earlier, many FSEDs operate as revenue centers and feeders to a main institution/hospital if a patient does require admittance.

The aim of this study is to retrospectively investigate and quantify the value of a scribe program in a highly efficient free-standing emergency department (FSED). Ultimately, the authors seek to answer if the implementation of scribes in the free-standing ED is a cost-effective decision. The authors hypothesize that the addition of scribe services to a freestanding ED will increase overall physician productivity and will lead to a positive impact on revenue, while improving throughput and increasing the value of care.

\section{Methods}

2.1. Study Setting and Population. The University of Florida Health FSED is situated in an 8,500-square-foot space, with all of the necessary technology and diagnostic resources for comprehensive emergency care, including on-site lab services, X-ray, ultrasound, and CT imaging. The department offers 10 treatment spaces and has a permanently stationed University of Florida Health ambulance to transfer any patients requiring admission to the main hospital. At the onset of the study period (December 2013), the FSED was staffed daily by two attending physicians on 12-hour shifts (7:00 a.m. to 7:00 p.m. and 7:00 p.m. to 7:00 a.m.). Since then, volume increases have led to an additional overlapping midlevel provider to provide clinical coverage from 11:00 a.m. to 11:00 p.m. While on shift, a scribe completes charting of the electronic medical record either in real-time (in the room) or via a dictation after the encounter. Scribes are encouraged to accompany a physician to every patient encounter, unless they are appreciably behind on their documentation.

Scribes are responsible for a patient's history of present illness, review of systems, and physical exam. In many cases, scribes can also review and synthesize a patient's medical history, input outside records, pull relevant imaging impressions into the note, and take dictations for a provider's interpretation of electrocardiogram, radiography, and/or laboratory results, in conjunction with their medical decision making and reevaluations. Scribes do not write orders per University of Florida Health's compliance office and Centers of Medicare \& Medicaid Services regulations.

2.2. Outcome Measures. The aim of the study was to assess the quantifiable value, in terms of revenue and in productivity that utilizing a scribe added to the FSED. Researchers aimed to assess this by measuring differences between scribe and nonscribe cohorts in the discrete, encounter-specific variables of the following:

(1) Charges: the professional fee charges from a patient encounter.

(2) Collections: the professional fee collections from a patient encounter.

(3) wRVUs: RVUs (relative value units) which are a structured scale by which physician services are counted. The portion of the service that measures the physician's work (wRVU) takes into consideration the time, skill, and effort that goes into a patient encounter. In the United States, wRVUs act as reliable and objectives measures of physician productivity (i.e., the higher the $\mathrm{wRVU}$ is, the more productive the physician is, and the more the reimbursement they received).

These measures were aggregated monthly as metrics (a) for the entire department and (b) for each individual patient. In both subsets, deidentified data was sorted by whether the encounter involved the assistance of a medical scribe. As this was examined on a per month basis, this meant aggregating all scribe versus nonscribe data for the time period of 11:00 a.m. to 9:00 p.m. for the entire month. As noted above, revenues are strictly being analyzed in terms of professional fee revenues only and not hospital fees. 
Researchers also set out to assess if scribes had any effect on FSED throughput. Throughput was assessed by using common ED metrics:

(1) Arrival-to-medical evaluation initiated: the time elapsed when a patient arrives in the ED until the provider is able to begin evaluating the patient. Another term for this is "door-to-doctor" or "doorto-provider" time.

(2) The number of patients who leave without being seen (LWBS).

(3) Total registered visits and total patients seen by the provider(s).

Researchers sought to discern any statistical differences in these measures during the 11:00 a.m. to 9:00 p.m. time period on days when a scribe was and was not on shift. Additional demographic data was obtained for each patient encounter to assess if the study was subject to any selection bias.

2.3. Study Design. This study was performed at the University of Florida Health Springhill Emergency Department (FSED). After receiving approval from the University of Florida Institutional Review Board (IRB), administrative data was collected retrospectively via the University of Florida Decision Support Services (DSS) and Faculty Practice Decision Support (FPDS) from the time period of December 1, 2013, to February 1, 2015. Measures of efficiency and productivity collected were charges/month, collections/month, wRVUs/month, charges/patient, collections/patient, and wRVUs/patient. Departmental throughput data collected included number of registered visits, number of patients seen, and number of patients who leave without being seen (LWBS). Additional throughput measures that were specific to patient encounters alone, such as ED arrival hour, and time from ED arrival-to-medical evaluation initiated (in minutes) were also obtained; this measure is commonly known as "door-to-doctor" time and carries implications for providing appropriate care in the setting of an emergency department. For throughput data such as numbers of LWBS, "door-to-doctor" time, total patient visits, and total registered visits, a scribe designation was given if a scribe was scheduled and completed a shift that day.

For efficiency and productivity data, such as charges, collections, and wRVUs, two SCRIBE flags were used. The first flag indicated if a scribe was assigned to the patient's treatment team. The second flag indicated if a scribe was scheduled and completed a shift that day. At the beginning of the study period, scribes were being utilized in the FSED on an intermittent basis, with focus given to days where the department had its highest volume. As time progressed, the scribe coverage for FSED providers grew. By February 2015, two scribes were being staffed in the FSED every day on overlapping shifts, permitting each of the two FSED providers (one attending and one midlevel) to utilize their own scribe. To maintain consistency, researchers decided to only examine encounters (ED arrival time) that occurred between 11:00 a.m. and 9:00 p.m. during the 15-month study period. This 10 -hour block was used to control any confounding variables between times of day when scribes were and were not on shift in the FSED. This block corresponds to the shift when scribes were initially assigned at the beginning of the study period.

2.4. Data Analysis. Both aims of this study were assessed using retrospective statistical analysis methods on scribe or nonscribe data from December 1, 2013, to February 1, 2015. Analysis was conducted with the assistance of the Decision Support Services (DSS) and Faculty Practice Decision Support (FPDS) divisions. Measures of efficiency and productivity were merged and plotted against the number of scribe shifts utilized per month in the FSED to assess variance (see Figure 1). Ultimately, average differences in scribe and nonscribe cohorts of charges, collections, and wRVUs were calculated. Throughput measures were also averaged and compared between scribe and nonscribe cohorts (see Table 1). Using payroll data and the known actual cost of scribe labor, analysis was performed to assess if the scribe is ultimately paying for himself or herself while bringing added value to the department. This data was computed using the software R: The R Project for Statistical Computing (https://www.r-project.org/).

\section{Results}

3.1. Throughput, Patient Volumes, and LWBS. During the study period, which comprised a total of 512 days, a scribe was present during the 11:00 a.m. to 9:00 p.m. time on 338 days (66\% of the time). Within this 10 -hour period on these days, 11,637 patients were treated. There were no scribes present on 174 days (34\% of the time). Within this 10 -hour period on these days, 3,803 patients were treated. As discussed in previous sections, scribes were added to the FSED in a nonuniform manner, resulting in an uneven distribution of scribe shifts versus nonscribe shifts. Nearly $70 \%$ of scribe shift days occurred after the midpoint of the study in July 2014. While this was also occurring, the FSED experienced a steady rise in monthly patient volumes. Monthly LWBS rates were relatively unchanged. Figure 2 tracks this trend over the course of the study period.

Ultimately, this data equates to the department treating on average 34.42 patients during the 10 -hour period when the scribe was present versus 21.86 patients during the same period when the scribe was not present. 95\% confidence intervals could not be calculated for patients per day because the data did not include date of arrival. Day-to-day variation could thus not be determined. In measures of throughput, on average, the arrival-to-medical evaluation initiated decreased by 2.74 minutes ( $95 \% \mathrm{CI}:-6.28<x<0.80$ ) on days when a scribe was present (see Table 1). Patients who left without being seen (LWBS) did not significantly change on days with a scribe present (see Figure 2). Worth noting, during the study period, roughly $68 \%$ of patients who arrived between 11:00 a.m. and 9:00 p.m. had a scribe which completes their chart.

Additional demographic data of the 11,637 patients were obtained, including age, gender, triage level, race, and ethnicity. Data is expressed in terms of percent. Between scribe and nonscribe patients, there was no appreciable difference in all demographics. Thus, the authors concluded that there was no 
TABLe 1

(a)

\begin{tabular}{lcccr}
\hline & Total days & Total patients treated & Patients treated per day & Arrival-to-MEI (minutes) \\
\hline Scribe shifts & 338 & 11,637 & 34.42 & 39.90 \\
Nonscribe shifts & 174 & 3,803 & 21.86 & 42.64 \\
Differences & 164 days & 7834 patients & 12.56 patients & -2.74 minutes \\
\hline
\end{tabular}

(b)

\begin{tabular}{lcc}
\hline Scribe? & & Gender \\
& Male & Female \\
\hline No & $37 \%$ & $63 \%$ \\
Yes & $37 \%$ & $63 \%$ \\
\hline
\end{tabular}

(c)

\begin{tabular}{|c|c|c|c|c|c|c|c|c|c|c|c|c|}
\hline \multirow{2}{*}{ Scribe? } & \multicolumn{12}{|c|}{ Age } \\
\hline & $0-10$ & $10-20$ & $20-30$ & $30-40$ & $40-50$ & $50-60$ & $60-70$ & $70-80$ & $80-90$ & $90-100$ & $>100$ & NA \\
\hline No & $6 \%$ & $10 \%$ & $22 \%$ & $19 \%$ & $14 \%$ & $12 \%$ & $8 \%$ & $5 \%$ & $3 \%$ & $1 \%$ & $0 \%$ & $0 \%$ \\
\hline Yes & $6 \%$ & $10 \%$ & $22 \%$ & $17 \%$ & $14 \%$ & $12 \%$ & $10 \%$ & $6 \%$ & $3 \%$ & $1 \%$ & $0 \%$ & $0 \%$ \\
\hline
\end{tabular}

(d)

Scribe?

Race

Unknown American Indian Asian Black Black Hispanic Hispanic Multiracial White White Hispanic Other

\begin{tabular}{|c|c|c|c|c|c|c|c|c|c|c|c|}
\hline No & $0 \%$ & $0 \%$ & $1 \%$ & $30 \%$ & $0 \%$ & $0 \%$ & $0 \%$ & $63 \%$ & $0 \%$ & $4 \%$ & $0 \%$ \\
\hline Yes & $0 \%$ & $0 \%$ & $1 \%$ & $28 \%$ & $0 \%$ & $0 \%$ & $0 \%$ & $66 \%$ & $0 \%$ & $4 \%$ & $0 \%$ \\
\hline
\end{tabular}

(e)

\begin{tabular}{|c|c|c|c|c|}
\hline \multirow{2}{*}{ Scribe? } & \multicolumn{4}{|c|}{ Ethnicity } \\
\hline & Hispanic & Not Hispanic & Patient refused & Unknown \\
\hline No & $4 \%$ & $95 \%$ & $0 \%$ & $0 \%$ \\
\hline Yes & $4 \%$ & $96 \%$ & $0 \%$ & $0 \%$ \\
\hline
\end{tabular}

(f)

\begin{tabular}{|c|c|c|c|c|c|c|}
\hline \multirow{2}{*}{ Scribe? } & \multicolumn{6}{|c|}{ Acuity } \\
\hline & ESI-1 & ESI-2 & ESI-3 & ESI-4 & ESI-5 & Unknown \\
\hline No & $0 \%$ & $11 \%$ & $51 \%$ & $35 \%$ & $2 \%$ & $1 \%$ \\
\hline Yes & $0 \%$ & $12 \%$ & $54 \%$ & $32 \%$ & $1 \%$ & $0 \%$ \\
\hline
\end{tabular}

evidence of any selection bias on the part of the scribes who were seeing patients.

3.2. Charges, Collections, and wRVUs. In terms of average charges per patient and collections per patient, scribed charts charged \$14.49 more (95\% CI: \$5.61<x<\$23.36) and collected $\$ 4.69$ more (95\% CI: $\$ 0.73<x<\$ 8.65$ ) than nonscribed counterparts. Scribed charts also had an average difference of 0.10 wRVUs/patient (95\% CI $0.05<x<0.16$ ) (see Table 2).

The per-patient metrics in Table 2 were plotted against the number of days of scribe coverage per month to assess a variance when a scribe was or was not on shift in the FSED (see Figure 1). The crosses represent an aggregation of all scribed charts over a monthly period, while the dots represent an aggregation of all nonscribed charts over the same period. Crosses and dots that share the same $x$-value occur during the same month.
To compare department totals on days where a scribe was and was not working, per-patient data from Table 2 was extrapolated. When a scribe was working, 34.42 patients were seen on average. When a scribe was not working, 21.86 patients were seen on average. In addition, data shows that scribes accounted for the charts of $68 \%$ of patients who arrived to the FSED on days when a scribe was present.

Alternatively, $32 \%$ of patients on these days were not charted by scribes. Thus, using the scribe chart and nonscribe chart data, we can calculate totals for a 10-hour scribe shift and a 10-hour nonscribe shift. These calculations can be found in Table 3. Of note, as mentioned in Section 2.3, scribes were added preferentially to days where the FSED would have higher patient volumes. Thus, researchers cannot assume that scribes had any effect on the number of patients seen per 10hour shift. It is for this reason that the calculations in Table 3 are not meant to represent the whole contribution that a 
Productivity metrics with scribed and nonscribed charts

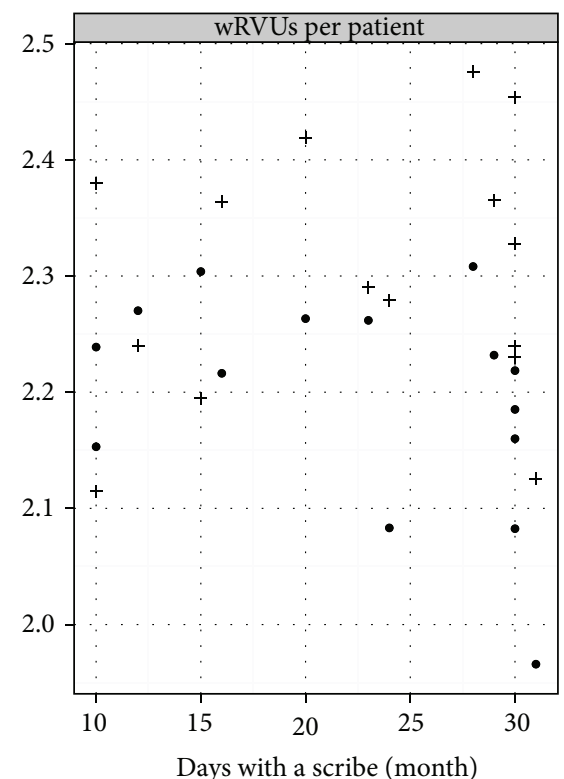

Scribe

- Nonscribe

+ Scribe

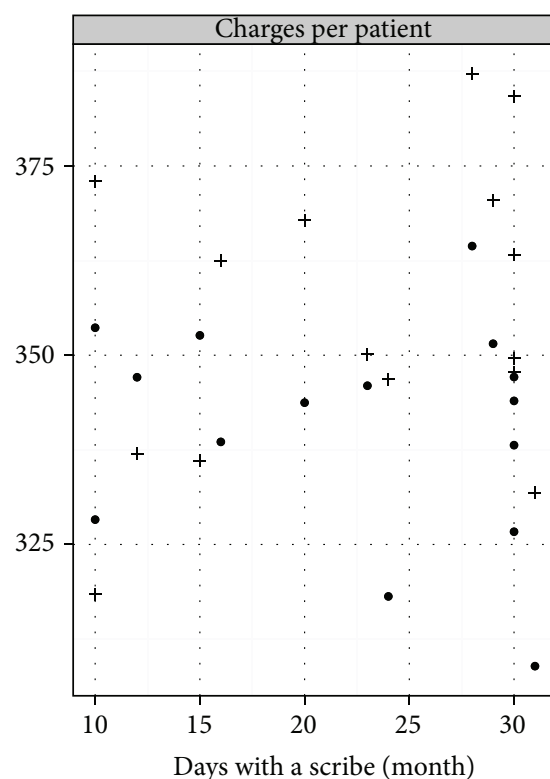

Scribe

- Nonscribe

+ Scribe

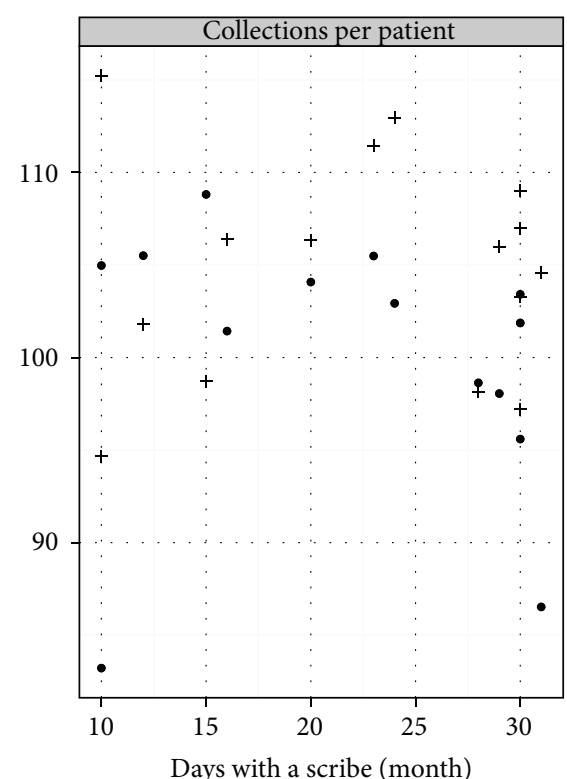

Scribe

- Nonscribe

+ Scribe

Figure 1

Emergency department monthly patient volumes Date range: $8 / 1 / 2013$ to $7 / 31 / 2015$

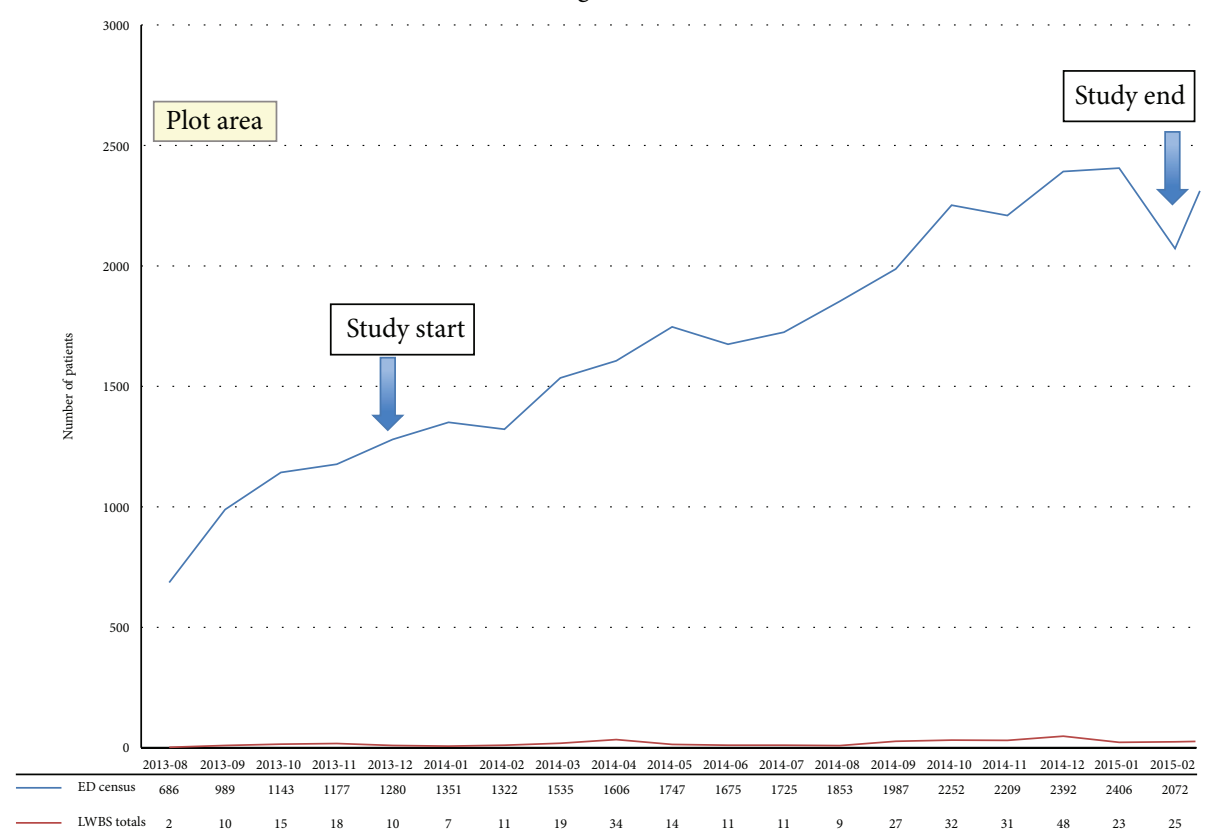

Figure 2

TABLE 2

\begin{tabular}{lccr}
\hline & Charges per patient & Collections per patient & wRVUs per patient \\
\hline Scribe charts & $\$ 355.07$ & $\$ 104.85$ & 2.3 \\
Nonscribe charts & $\$ 340.58$ & $\$ 100.16$ & 2.2 \\
Increase from scribe charts & $\$ 14.49$ & $\$ 4.69$ & 0.1 \\
$\%$ increase from scribe charts & $4.25 \%$ & $4.68 \%$ & $4.55 \%$ \\
\hline
\end{tabular}


TABLE 3

(a)

\begin{tabular}{lccc}
\hline Charges & Charges per patient & Charges per shift & Charges per hour \\
\hline Scribe shifts & $355.07 /$ scribe chart & $\$ 12,061.91$ & $\$ 1,206.19$ \\
Nonscribe shifts & $340.58 /$ nonscribe chart & $\$ 7,445.08$ & $\$ 744.51$ \\
Difference between scribe shifts and nonscribe shifts & $\$ 14.49$ & $\$ 4,616.83$ & $\$ 461.68$ \\
\hline
\end{tabular}

(b)

\begin{tabular}{lccc}
\hline Collections & Collections per patient & Collections per shift & Collections per hour \\
\hline Scribe shifts & $\$ 104.85 /$ scribe chart & $\$ 3,557.28$ & $\$ 355.73$ \\
Nonscribe shifts & $\$ 100.16 /$ nonscribe chart & $\$ 2,189.50$ & $\$ 218.95$ \\
Difference between scribe shifts and nonscribe shifts & $\$ 4.69$ & $\$ 1,367.78$ & $\$ 136.78$ \\
\hline
\end{tabular}

(c)

\begin{tabular}{lccc}
\hline wRVUs & wRVUs per patient & wRVUs per shift & wRVUs per hour \\
\hline Scribe shifts & $2.3 /$ scribe chart & 78.06 & 7.81 \\
Nonscribe shifts & $2.2 /$ nonscribe chart & 48.09 & 4.81 \\
Difference between scribe shifts and nonscribe shifts & 0.1 & 29.97 & 3.00 \\
\hline
\end{tabular}

scribe makes to the FSED. The estimated added contribution of hiring a scribe will be discussed in the next section.

\subsection{Incremental Cost-Effectiveness Ratio and Return on Invest-} ment Calculations. To assess whether the implementation of scribes represents a valuable decision in the setting of the free-standing emergency department, incremental costeffectiveness ratios and return on investment can be calculated.

For the incremental cost-effectiveness ratio, the cost of the intervention (adding scribes) is placed in the numerator and the effect of the intervention is placed in the denominator:

$$
\mathrm{ICER}_{\text {Scribes }}=\frac{C_{1}-C_{0}}{E_{1}-E_{0}} .
$$

In these equations, $C_{1}$ is chosen to represent the average cost of a full-time scribe $(\$ 10.50)$ and a part-time scribe (\$8.25) per hour: $\$ 9.375 . C_{0}$ is $\$ 0$, as it represents the case where a scribe is not working. The effect of the intervention can be examined in terms of accessibility metrics and later extrapolated to discuss improving the value of care.

The other calculation that can be made is important from the department's standpoint. The return on investment seeks to answer whether hiring scribes is a fiscally prudent decision. If scribes can generate revenue to pay for their wage, while also providing proven benefits to the department and to physicians, then their implementation is supported.

3.3.1. Accessibility Metrics. This is measured in average doorto-medical evaluation initiated:

$$
\begin{aligned}
& C_{1}=\$ 9.375, \\
& C_{0}=\$ 0, \\
& E_{1}=39.90 \text { minutes, } \\
& E_{0}=42.64 \text { minutes, }
\end{aligned}
$$

$$
\begin{aligned}
\mathrm{ICER}_{\text {Scribes }} & =\frac{\$ 9.375-0}{39.90-42.64 \text { minutes }} \\
& =-3.33 \$ / \text { minutes. }
\end{aligned}
$$

3.3.2. Return on Investment. This equation is simply a ratio between the financial gain less the cost and over the cost. The investment is the decision to implement a 10-hour scribe shift in the free-standing emergency department. The initial cost to train and hire a scribe is omitted and negligible:

$$
\begin{aligned}
& \text { ROI }_{\text {Scribe }} \\
& =\frac{\text { Gain from Investment }- \text { Cost of Investment }}{\text { Cost of Investment }} .
\end{aligned}
$$

To establish the gain from investment, we must first perform the following calculation, with an important assumption. We assume that scribes had no effect on the number of patients treated per shift. In other words, on a day where a scribe was working and 34.42 patients were seen, we assume that the same 34.42 patients who would also be seen had the scribe which had not been present. The only added value thus comes from the fact that scribes charge and collect more per chart. In this case, an average shift with scribes brings in an additional $\$ 33.92$ in charges per hour and $\$ 10.95$ in collections per hour. The ROI is thus calculated to be $\$ 2.62$ in charges per hour and $\$ 0.17$ in collections per hour:

$$
\begin{aligned}
& \$ 14.49 \frac{\text { added charges }}{\frac{\text { scribe patient }}{\text { scribe shift }}} \times 34.42 \frac{\text { total patients }}{\text { scribe }} \\
& \times 0.68 \frac{\text { scribe patient }}{\frac{\text { total patients }}{\text { charges }}}=\$ 339.15 \frac{\text { added charges }}{\text { scribe shift }} \\
& =\$ 33.92 \frac{\text { hour }}{\text { hour }}
\end{aligned}
$$




$$
\begin{aligned}
& \$ 4.69 \frac{\text { added collections }}{\text { scribe patient }} \times 34.42 \frac{\text { total patients }}{\text { scribe shift }} \\
& \times 0.68 \frac{\text { scribe patient }}{\frac{\text { total patients }}{\text { collections }}}=\$ 109.54 \frac{\text { added collections }}{\text { scribe shift }} \\
& =\$ 10.95 \frac{\text { hour }}{} \\
& \mathrm{ROI}_{\text {Scribe Charges }}=\frac{\$ 33.92-\$ 9.375}{\$ 9.375}=\$ 2.62 / \text { hour, } \\
& \mathrm{ROI}_{\text {Scribe Collections }}=\frac{\$ 10.95-\$ 9.375}{\$ 9.375}=\$ 0.17 / \mathrm{hour} .
\end{aligned}
$$

The authors conclude that the decision to implement a scribe in the University of Florida Health free-standing emergency department is fiscally prudent because the revenue generated by scribes' efforts offsets their cost in wage.

\section{Discussions}

4.1. Throughput, Patient Volumes, and LWBS. One aim of this study was to assess if scribes had any effect on FSED throughput. The data demonstrates that while providers treated 1.25 more patients per hour on scribe shift days versus nonscribe shift days, patients were able to see a provider 2.74 (95\% CI: $-6.28<x<0.80)$ minutes faster when a scribe was present. As prior studies have illustrated that nearly $44 \%$ of EM physicians time spent on shift is dedicated to EMR entry, having a scribe present allows physicians to decrease this task significantly [2].

Though differences in LWBS were not statistically significant, it is important to mention that in the 24 months since the University of Florida Health FSED's opening, the LWBS rate has remained steady at $1.09 \%$ (see Figure 2). In addition, the University of Florida Health FSED has seen patient volumes grow from 686 in August 2013 to 2581 in July 2015. More specifically, the mean patient per month average prior to the start of the study period (from AugustNovember 2013) in the FSED was roughly 999 patients per month. In the 15-month period of the study (December 2013February 2015), the FSED averaged more than twice the prestudy average: roughly 2008 patients per month.

The effect that scribes had on the LWBS rate is difficult to assess. While arrivals in the FSED increased nearly $62 \%$ since it initially opened in August 2013, there have been other notable changes in department staffing, such as adding midlevel providers, nurses, and technicians. Even before scribes were added, there was no appreciable difference in LWBS.

4.2. Cost-Effectiveness and Value of Care. The results of this study concluded that scribes increased revenue and also improved FSED throughput by a statistically significant outcome of 2.74 (95\% CI: $-6.28<x<0.80)$ minutes. This finding is noteworthy because it contributes to better patient outcomes, which is supported by literature that demonstrates an inverse relationship between patient wait time and quality of care. In 2009 study published in the Annals of Emergency Medicine concluded that increased emergency department (ED) wait time reduced quality of care and increased adverse events [8]. This universal relationship is also evident in a study published by the Department of Emergency Medicine at the American University of Beirut Medical Center in September 2015; when implementing lean methodology in an attempt to decrease door-to-doctor time there was improvement in other ED operations. Using the lean methodology door-to-doctor times decreased by $37 \%$ when comparing preand postinterventions. That study also found that there was a reduction in mean ED length of stay in both admitted and discharged patients, thus improving patient outcomes [9]. Although this data is not reflected in the concentration of our study, one can conclude that since scribes decrease door-todoctor time we can expect improved patient outcomes. Given these results we can reach the conclusion that scribes in the FSED increase value of care and patient outcomes given that they decrease door-to-doctor time.

4.3. Implications on Existing Literature Evaluating Scribes in the ED. As the transition to the electronic medical record has only occurred in the last decade, the adoption of scribe programs is a very recent activity in the healthcare landscape. Literature supporting scribe usage in emergency departments is limited, but growing. A common scribe metric studied is physician satisfaction. At the University of Florida, physicians in the adult emergency department were more satisfied after the implementation of scribes [4]. Further considerations for this are discussed in the next section. Other metrics previously studied include the same data collected in this study, including metrics of productivity, throughput, and charges $[1,8,9]$.

In 2006-2007-study examining 243 clinical shifts in a university-based academic emergency medicine department (census of 58,000 visits annually), RVUs increased by 0.24 units (95\% CI: $0.10<x<0.38$ ) per hour for every $10 \%$ increment in scribe usage [1]. In a 2010-study in a suburban emergency department (census of 78,000 visits annually), using the same electronic medical record system as this study, "door-to-doctor" time decreased by 13 minutes postscribe implementation (no 95\% CI provided, $P<0.0001$ ) [10]. Lastly, in a recently published study within academic emergency medicine department (with almost 160,000 patient records reviewed over one year), scribed charts had an increase in \$44.31 in charges (95\% CI: \$44.30<x<\$44.32) per chart [11].

While these studies offer support for the implementation of scribes into academic and suburban emergency departments, to the authors of this study's knowledge, there is no literature on scribes in the setting of a free-standing emergency department. In addition, this study is unique in that it assesses the cost-effectiveness of scribes by using information about a scribe's wage.

4.4. Other Factors and Considerations. An additional added value of scribes, not previously discussed, is the indirect benefits of physician satisfaction. While there are many factors involved in physician turnover, dissatisfaction is 
associated with higher rates of turnover [10]. The University of Florida Health ED Scribe Program has already been shown to increase provider satisfaction in the adult emergency department, and keeping providers happy and less stressed at work can also benefit the department [4]. For one, the issue of physician turnover has the potential to impact a hospital through lost productivity, recruiting costs, and decreased morale among the remaining staff [11]. On the subject of turnover, from a purely financial standpoint, practices should expect to spend between $\$ 20,000$ and $\$ 30,000$ to recruit a physician if hiring via an independent firm [12]. This cost, plus lost income and other physician startup costs, can be substantial [13].

\section{Limitations}

Many of the limitations of this study have previously been mentioned. One limitation is that only $68 \%$ of all patients were seen by a scribe. Therefore, some variance could exist between the reported revenue generated per patient and the true value. Also scribes were added to the FSED in a nonuniform manner to accommodate increasing FSED volumes. In addition, the program benefitted from multiple hiring classes, which increased the number of scribes that were trained and able to staff a shift in the FSED. As the scribe services were increasing, scribe shifts were scheduled preferentially on the busiest days for the FSED, which may lead to some overestimation of the revenue associated with scribe service. $70 \%$ of the scribe shifts occurred after the midpoint of the study (July, 2014).

A third limitation is that the FSED physicians have a compensation plan that in part is based on their personal LWBS and time from arrival to physician metrics. While scribes certainly have improved these measures, there are also other competing factors in terms of bonus compensation which influence physician behavior to minimize these metrics.

As previously mentioned, the determination of whether a scribe was assigned to the record or not depended wholly on the scribe entering that information in the EHR. On days with just one scribe, it may not have been intuitive for a scribe to always make that indication since they were the only scribe present, thus underestimating their productivity for the day. There was no subanalysis of patient acuity and chart complexity. While total wRVUs are one measure of productivity, it does not necessarily account for the amount of time spent per scribe on each chart. Finally, this study took place in one FSED associated with an academic health system. Further study would be needed to see if these results carry over to other independent FSEDs.

\section{Conclusions}

This study was designed to look at the value of the addition of scribes to a FSED with regard to throughput and financial measures. The impact of a scribe program on the operational metric of door-to-provider time was small but statistically significant. On the surface, the scribe program did not appear to have a significant impact on the left without being seen rate. However, it was noted that the overall patient volume of this FSED increased by $62 \%$ during the study period, and it is possible that both of these operational metrics could have had negative results without the presence of scribes. Further study is needed at a more mature FSED with stable volumes to determine the true impact of scribes on these operational metrics.

The financial impact of scribes at a FSED is more robust and shows that the implementation of scribes results in a net increase of revenue and collections even with conservative measures and analysis. Coupled with this financial gain was a net increase in per-provider productivity shown via increased wRVUs.

The authors conclude that implementing a scribe program at a FSED is cost-effective and justified from both an operational and a financial analysis.

\section{Conflict of Interests}

The authors declare that there is no conflict of interests regarding the publication of this paper.

\section{References}

[1] R. Arya, D. M. Salovich, P. Ohman-Strickland, and M. A. Merlin, "Impact of scribes on performance indicators in the emergency department," Academic Emergency Medicine, vol. 17, no. 5, pp. 490-494, 2010.

[2] R. G. Hill Jr., L. M. Sears, and S. W. Melanson, “4000 Clicks: a productivity analysis of electronic medical records in a community hospital ED," The American Journal of Emergency Medicine, vol. 31, no. 11, pp. 1591-1594, 2013.

[3] C. G. Shultz and H. L. Holmstrom, "The use of medical scribes in health care settings: a systematic review and future directions," Journal of the American Board of Family Medicine, vol. 28, no. 3, pp. 371-381, 2015.

[4] B. Allen, B. Banapoor, E. C. Weeks, and T. Payton, "An assessment of emergency department throughput and provider satisfaction after the implementation of a scribe program," Advances in Emergency Medicine, vol. 2014, Article ID 517319, 7 pages, 2014.

[5] “Scribes, EMR please docs, save \$600,000," ED Management, vol. 21, no. 10, pp. 117-118, 2009.

[6] A. Scheck, "The next big thing: medical scribes: scribes push emergency medicine closer to adoption of electronic medical records," Emergency Medicine News, vol. 31, no. 2, pp. 13-16, 2009.

[7] A. Ayers and J. Nawlaniec, Freestanding Emergency Departments Earn Traction, vol. 2, Urgent Care Association of America, Naperville, Ill, USA, 2012.

[8] L. I. Horwitz, B. A. Jeremy Green, and E. H. Bradley, "US emergency department performance on wait time and length of visit," Annals of Emergency Medicine, vol. 55, no. 2, pp. 133-141, 2010.

[9] M. J. El Sayed, G. R. El-Eid, M. Saliba, R. Jabbour, and E. A. Hitti, "Improving emergency department door to doctor time and process reliability: a successful implementation of lean methodology," Medicine, vol. 94, no. 42, Article ID e1679, 2015.

[10] A. Bastani, B. Shaqiri, K. Palomba, D. Bananno, and W. Anderson, "An ED scribe program is able to improve throughput time and patient satisfaction," American Journal of Emergency Medicine, vol. 32, no. 5, pp. 399-402, 2014. 
[11] N. Gupta, M. Kopp, and B. M. Becker, "247 scribes in an academic emergency department lead to increased charges and decreased down coding," Annals of Emergency Medicine, vol. 64, no. 4, article S88, 2014.

[12] S. B. Buchbinder, M. Wilson, C. F. Melick, and N. R. Powe, "Primary care physician job satisfaction and turnover," American Journal of Managed Care, vol. 7, no. 7, pp. 701-713, 2001.

[13] W. J. Meurer, C. B. Sozener, Z. Xu et al., "The impact of emergency physician turnover on planning for prospective clinical trials," The Western Journal of Emergency Medicine, vol. 14, no. 1, pp. 16-22, 2013. 


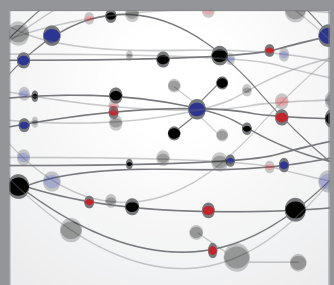

The Scientific World Journal
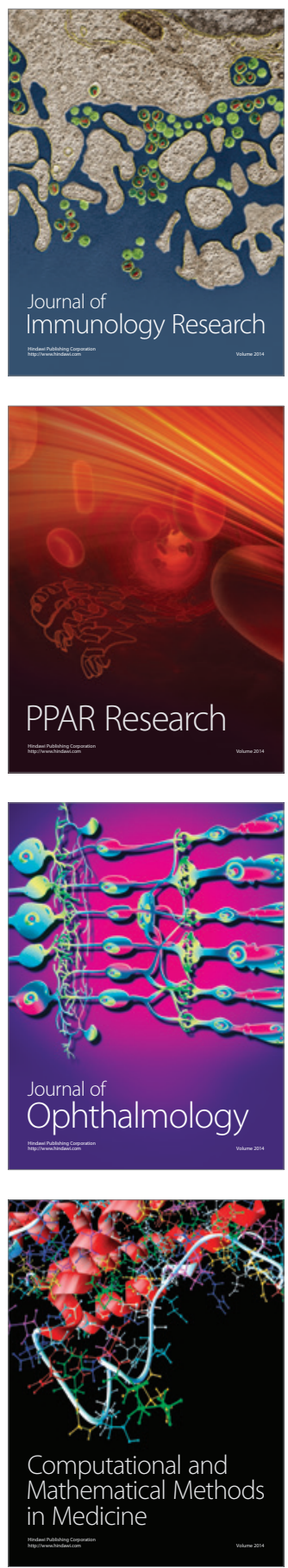

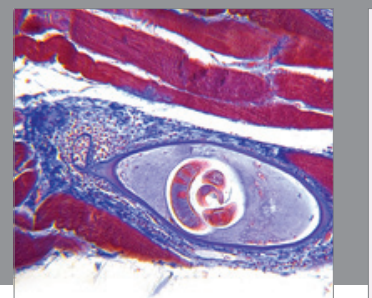

Gastroenterology

Research and Practice
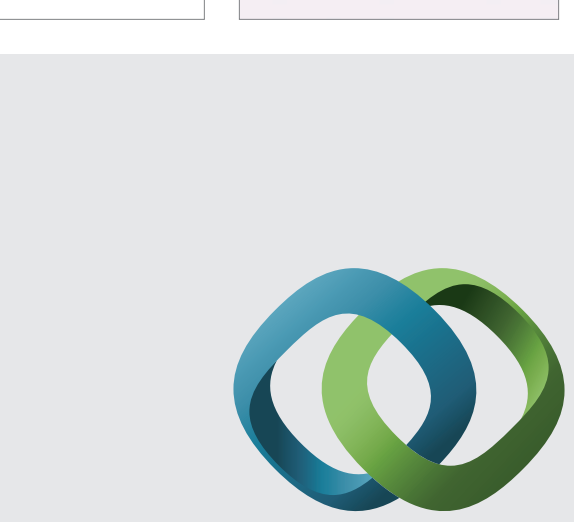

\section{Hindawi}

Submit your manuscripts at

http://www.hindawi.com
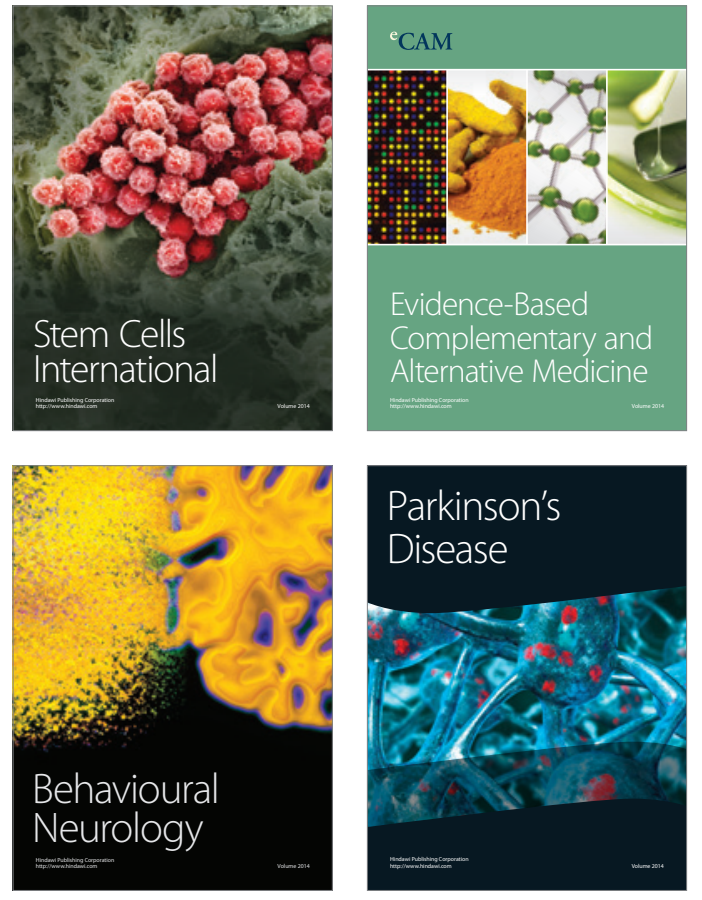
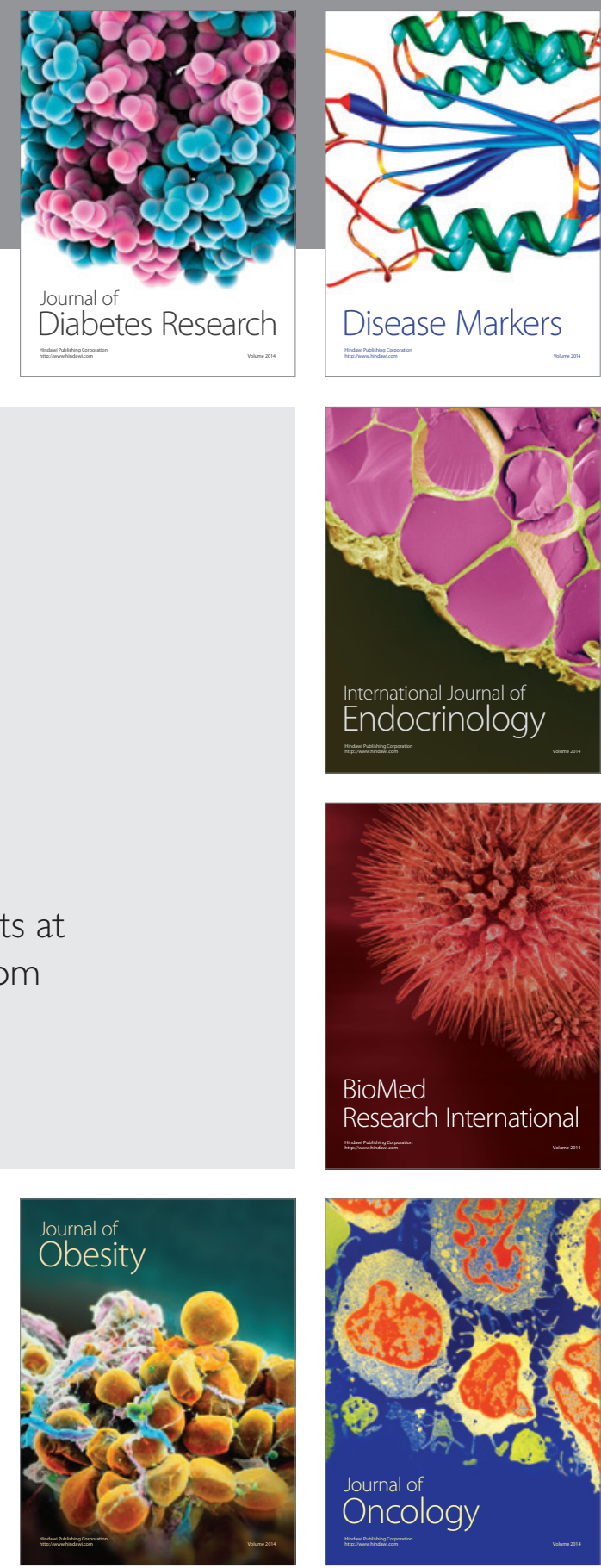

Disease Markers
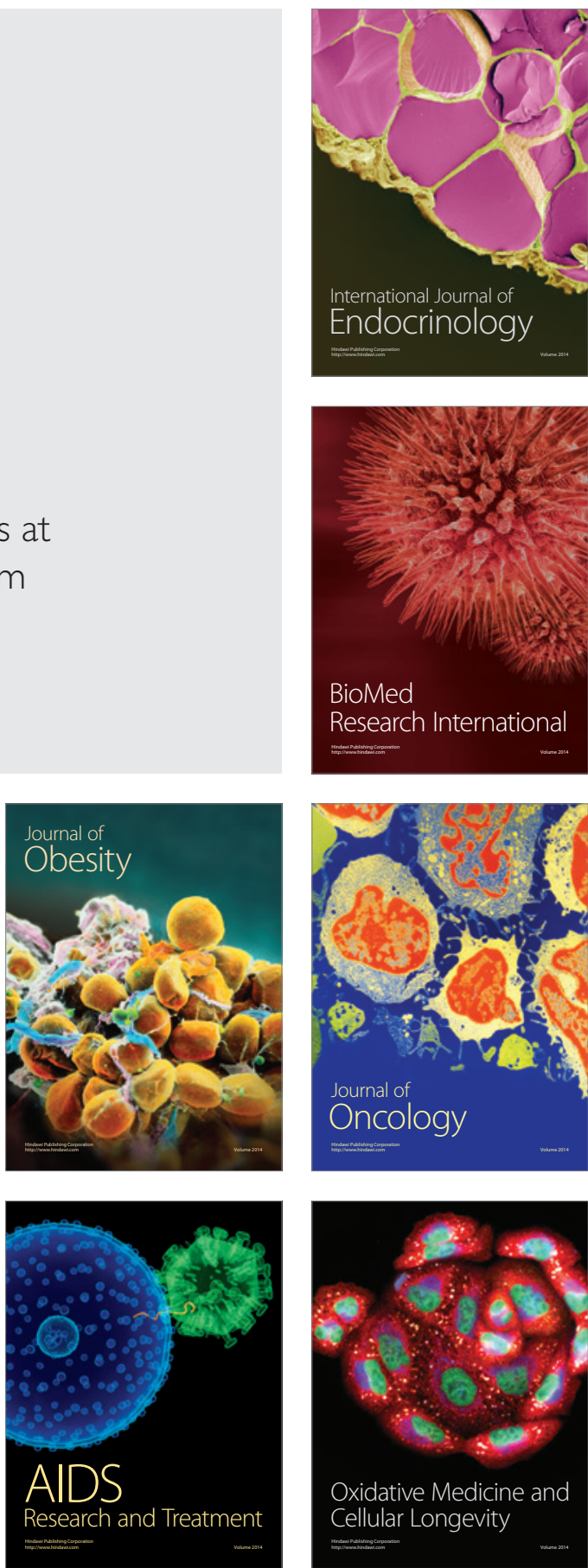